\title{
SYNTHESIS OF SEVERAL CHIRAL PHOSPHINAMIDES AND THEIR APPLICATION IN THE ASYMMETRIC BORANE REDUCTION OF PROCHIRAL KETONES
}

\author{
Kangying Li, Zhenghong Zhou, Lixin Wang, Qilin Zhou, and Chuchi Tang* \\ State Key Laboratory of Elemento-Organic Chemistry, Institute of Elemento-Organic Chemistry, Nankai \\ University, Tianjin 300071, P. R. China <c.c.tang@eyou.com>
}

\begin{abstract}
Several chiral phosphinamide catalysts were prepared from (S)-(+)-Betti Base, (D)-I R,2R-(-)-threo-I(p-Nitrophenyl)-2-amino-3-triphenyl-methoxypropanol-I and L-Serine respectively. Their application in the asymmetric borane reduction of prochiral ketones was examined. The chiral secondary alcohols were obtained in excellent chemical yields with moderate enantioselective excesses.
\end{abstract}

\section{INTRODUCTION}

The enantioselective reduction of prochiral ketones is an important route for the synthesis of optically active secondary alcohols ${ }^{[1]}$ which were widely used in the preparation of bioactive substances ${ }^{[2]}$. The enantioselevtive borane reduction catalyzed by oxazaborolidine prepared from chiral amino alcohols is one of the most efficient method ${ }^{[3]}$ which give excellent ee values and often have wide substrate scope. Other catalysts such as chiral sulfur reagents ${ }^{\left[{ }^{5} s_{1}\right]}$ and chiral phosphorous reagents ${ }^{[5]}$ were also used in this reaction. In recent years, great progress was achieved using chiral phosphorous reagents as catalysts for the reduction of prochiral ketones. In this paper, we reported several chiral phosphinamides compounds I, II and III prepared from (S)-(+)-Betti Base, (D)-IR,2R-(-)-threo-1-(p-Nitrophenyl)-2-amino-3-triphenylmethoxypropanol-I and L-Serine respectively. Their application as ligand catalysts in the asymmetric borane reduction of prochiral ketones were investigated.

\section{MATERIALS AND METHODS}

${ }^{1} \mathrm{H} N M R$ and ${ }^{31} \mathrm{P}$ NMR were recorded in $\mathrm{CDCl}_{3}$ as solvent on an AC-P200 instrument using TMS as internal standard and $85 \% \mathrm{H}_{3} \mathrm{PO}_{4}$ as external standard, respectively. Elemental analyses were done on an MF-3 automatic analyzer. Melting points were determined on an MP-500 melting point apparatus. Optical rotations were measured on a Perkin-Elmer 24IMC polarimeter. All temperatures were uncorrected.

Preparation of I-( $\alpha$-N-diphenylphosphinylaminobenzyl)-2-naph thol I (Scheme 1)

Scheme I<smiles>N[C@H](c1ccccc1)[C@H]1Cc2ccccc2CC1O</smiles>

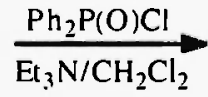

(S)-(+)-Betti Base 1<smiles>O=[PH+]([NH2+]C(c1ccccc1)c1ccccc1)c1ccccc1</smiles>

$0.757 \mathrm{~g}(3.2 \mathrm{mmol})$ diphenylphosphinyl chloride in $5 \mathrm{ml}$ dry $\mathrm{CH}_{2} \mathrm{Cl}$, was added to the solution of

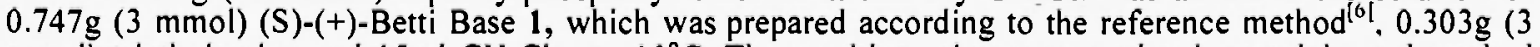

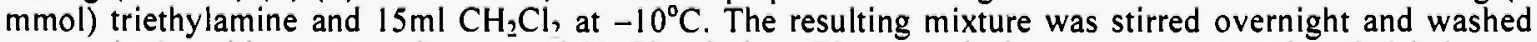
successively with water and saturated $\mathrm{NaCl}$ solution. The organic layer was separated and dried over anhydrous $\mathrm{Na}_{2} \mathrm{SO}_{4}$. After removal of the solvent the crude product was purified by column chromatography on silica gel (200-300 mesh, petroleum ether/EtOAc as eluent) to afford $0.47 \mathrm{~g}$ product as a white solid. Yield $35.0 \%$, m.p. $142 \sim 144^{\circ} \mathrm{C},[]_{0}=+57.0\left(\mathrm{c} 0.56, \mathrm{CH}, \mathrm{Cl}_{2}\right)$, ${ }^{31} \mathrm{P}$ NMR $26.37 \mathrm{ppm}(\mathrm{s})$. ' $\mathrm{H}$ NMR $2.15(\mathrm{~s}, \mathrm{H}, \mathrm{CH})$. 5.90 (bs, H, OH), 6.15 (s, lH, NH), $7.08 \sim 7.82\left(\mathrm{~m}, 2 \mathrm{lH}\right.$, Ar-H). Anal. Calcd for $\mathrm{C}_{29} \mathrm{H}_{24} \mathrm{NO}_{2} \mathrm{P}: \mathrm{C}, 77.49 ; \mathrm{H}$, $5.38 ; \mathrm{N}, 3.12$. Found: C, 77.54; H, 5.29; N, 3.13.

Preparation of (D)-I R,2R-(-)-threo-I-(p-Nitrophenyl)-2-(N-diphenylphosphinyl)amino-3-triphenylmethoxypropanol-1 II (Scheme 2)

To a solution of $1.14 \mathrm{~g}(2.5 \mathrm{mmol})$ (D)- I R,2R-(-)-threo-l-(p-Nitrophenyl)-2-amino-3-triphenylmethoxypropanol-1 2, which was prepared according to the reference method ${ }^{[7 !}$. and $0.5 \mathrm{lg}\left(5 \mathrm{mmol}^{2}\right)$ triethvlamine in $20 \mathrm{ml}$ dry $\mathrm{CH}_{2} \mathrm{Cl}_{2}$ was added $0.71 \mathrm{~g}(3 \mathrm{mmol})$ diphenylphosphinyl chloride in $10 \mathrm{ml} \mathrm{CH}_{2} \mathrm{Cl}_{2}$ under $-10^{\circ} \mathrm{C}$. The 
resulting mixture was warmed to room temperature and stirred overnight, and then washed successively with water and saturated $\mathrm{NaCl}$ solution. The organic layer was separated and dried over anhydrous $\mathrm{Na}_{2} \mathrm{SO}_{4}$.

\section{Scheme 2}<smiles>N[C@H](CC(=O)O)[C@H](O)[C@H](O)CCONc1ccccc1</smiles>

D-(-)-th reo-

2

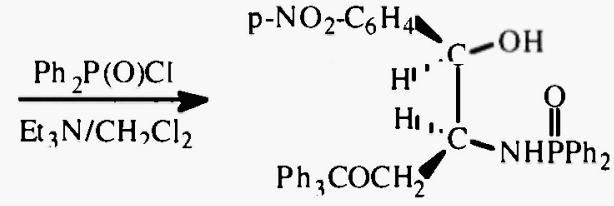

II

After removal of the solvent the crude product was purified by column chromatography on silica gel (200-300 mesh, petroleum ether/EtOAc as eluent) to give $0.5 \mathrm{~g}$ light yellow solid. Yield $30.7 \%$, m.p. $204 \sim 206^{\circ} \mathrm{C}$, [] ${ }_{\mathrm{D}}{ }^{2 \mathrm{v}}-43.7$ (c $\left.1.0, \mathrm{CH}_{2} \mathrm{Cl}_{2}\right),{ }^{31} \mathrm{P} \mathrm{NMR} 26.79 \mathrm{ppm}(\mathrm{s}),{ }^{1} \mathrm{H} \mathrm{NMR} 1.81 \sim 1.47\left(\mathrm{~m}, 2 \mathrm{H}, \mathrm{CH}_{2}\right)$, $1.89 \sim 2.15(\mathrm{~m}, 2 \mathrm{H}, \mathrm{CH}), 1.81 \sim 1.47\left(\mathrm{~d}, 6 \mathrm{H}, 2 \mathrm{CH}_{3}\right), 2.41 \sim 3.04\left(\mathrm{~m}, 2 \mathrm{H}, \mathrm{CH}_{2}\right), 4.68 \sim 4.80(\mathrm{~m}, 1 \mathrm{H}, \mathrm{CH}), 5.60$ (bs, $1 \mathrm{H}, \mathrm{OH}), 7.03 \sim 7.70(\mathrm{~m}, 18 \mathrm{H}, \mathrm{Ph}-\mathrm{H})$. Anal. Calcd for $\mathrm{C}_{40} \mathrm{H}_{35} \mathrm{~N}, \mathrm{O}_{5} \mathrm{P}: \mathrm{C}, 73.38 ; \mathrm{H}, 5.39 ; \mathrm{N}, 4.28$. Found: C, $73.19 ; \mathrm{H}, 5.20 ; \mathrm{N}, 4.17$.

Preparation of N-Triphenylmethyl-O-para-toluenesulphonyl-(2S)-serine methyl ester 4 and (-)-(2S)Methyl-1-tritylaziridine-2-carboxylate 5 : (Scheme 3)

These two compounds were prepared according to the procedures reported beforel ${ }^{8]}$.

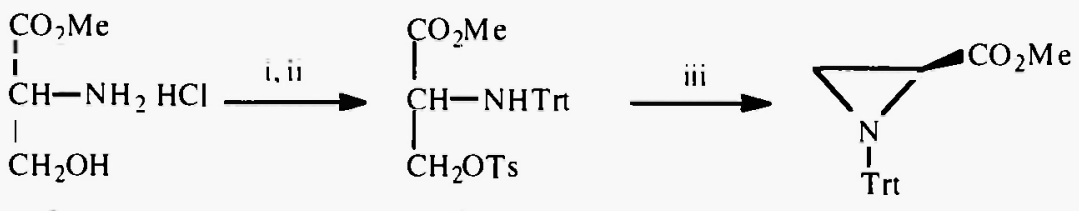

3

4

5

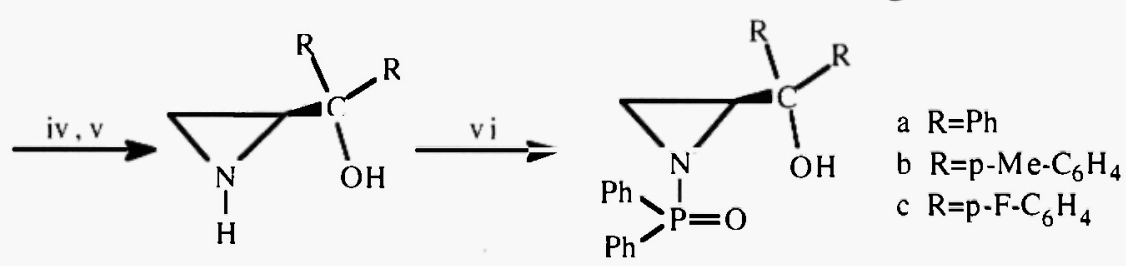

6

III

i) $\mathrm{Ph}_{3} \mathrm{CCl}, \mathrm{Et}_{3} \mathrm{~N}, \mathrm{CH}_{2} \mathrm{Cl}$; ii) $\mathrm{TsCl}$, Pyridine; iii) $\mathrm{Et}_{3} \mathrm{~N}$, THF, reflux; iv) $\mathrm{RMgBr}$, THF; v) $\mathrm{MeOH} / \mathrm{THF}$, $35 \% \mathrm{KOH}$; vi) $\mathrm{Ph}, \mathrm{P}(\mathrm{O}) \mathrm{Cl}, \mathrm{Et}_{3} \mathrm{~N}, \mathrm{CH}, \mathrm{Cl}_{2}$.

Preparation of (-)-(2S)-I-aziridin-2-yl(diaryl)methanol 6: according to the modified procedures reported by Zwanenburg ${ }^{l}$ ? .

(-)-(2S)-I-aziridin-2-yl(diphenyl)methanol 6a:

white solid, m.p. $158 \sim 160^{\circ} \mathrm{C}$, [] ${ }^{20}-18.3\left(\mathrm{c} 0.875, \mathrm{CH}_{2} \mathrm{Cl}_{2}\right),{ }^{1} \mathrm{H}$ NMR1.47 1.49 (d, $\left.2 \mathrm{H}, \mathrm{CH}_{2}\right), 2.72(\mathrm{~s}$, $1 \mathrm{H}, \mathrm{NH}), 3.32(\mathrm{~s}, 1 \mathrm{H}, \mathrm{OH}), 5.20(\mathrm{~s}, 1 \mathrm{H}, \mathrm{CH}), 7.18 \sim 7.47(\mathrm{~m}, 10 \mathrm{H}, \mathrm{Ph}-\mathrm{H})$. Anal. Calcd for $\mathrm{C}_{15} \mathrm{H}_{15} \mathrm{NO}: \mathrm{C}$, $79.97 ; \mathrm{H}, 6.71 ; \mathrm{N}, 6.22$. Found: $\mathrm{C}, 79.82 ; \mathrm{H}, 7.05 ; \mathrm{N}, 6.15$.

(-)-(2S)-I-aziridin-2-yl[di(p-methyl)-phenyl]methanol 6b:

white solid, m.p. $140 \sim 141^{\circ} \mathrm{C}$, [ ] ${ }_{\mathrm{D}}^{20}-15.2\left(\mathrm{c} \mathrm{l} 1.05, \mathrm{CHCl}_{3}\right),{ }^{1} \mathrm{H}$ NMR $1.25 \sim 1.27\left(\mathrm{~m}, 2 \mathrm{H}, \mathrm{CH}_{2}\right), 2.31$ (s,

$\left.6 \mathrm{H}, 2 \mathrm{CH}_{3}\right), 3.70 \sim 3.85(\mathrm{~m}, 2 \mathrm{H}, \mathrm{NH}, \mathrm{OH}), 4.85(\mathrm{~s}, \mathrm{HH}, \mathrm{CH}), 7.07 \sim 7.80(\mathrm{~m}, 8 \mathrm{H}, \mathrm{Ph}-\mathrm{H})$. Anal. Calcd for $\mathrm{C}_{17} \mathrm{H}_{19} \mathrm{NO}: \mathrm{C}, 80.59 ; \mathrm{H}, 7.56 ; \mathrm{N}, 5.53$. Found: $\mathrm{C}, 80.46 ; \mathrm{H}, 7.42 ; \mathrm{N}, 5.43$.

(-)-(2S)-I-aziridin-2-yl[di(p-fluoro)-phenyl|methanol 6c: light yellow solid, m.p. 92 94 ${ }^{\circ} \mathrm{C}$, [1] ${ }^{20}-18.5\left(\mathrm{c} 1.08, \mathrm{CHCl}_{3}\right),{ }^{1} \mathrm{H}$ NMR ${ }^{1} \mathrm{H}$ NMR $2.99 \sim 2.10(\mathrm{~m}, 2 \mathrm{H}$, $\left.\mathrm{CH}_{2}\right), 3.02 \sim 4.95(\mathrm{~m}, 3 \mathrm{H}, \mathrm{NH}, \mathrm{OH}, \mathrm{CH}), 7.18 \sim 7.47(\mathrm{~m}, 8 \mathrm{H}, \mathrm{Ph}-\mathrm{H})$. Anal. Calcd for $\mathrm{C}_{15} \mathrm{H}_{13} \mathrm{~F}, \mathrm{NO}: \mathrm{C}, 68.96$; H, 5.02; N, 5.36. Found: C, 69.13; H, 5.42; N, 5.10.

Typical procedure for praparation of compound IIIa c:

To a mixture of $2.3 \mathrm{mmol} 6,3 \mathrm{mmol}(0.303 \mathrm{~g}) \mathrm{Et}_{3} \mathrm{~N}$ and $15 \mathrm{ml} \mathrm{CH}, \mathrm{Cl}$, was added a solution of $2.5 \mathrm{mmol}$

$(0.59 \mathrm{lg}) \mathrm{Ph}, \mathrm{P}(\mathrm{O}) \mathrm{Cl}$ in $5 \mathrm{ml} \mathrm{CH}, \mathrm{Cl}$, under ice-salt bath. White precipitate was formed quickly, and the 
reaction was complete after the addition (monitored by TLC). The solid was collected by filtration and washed with $\mathrm{CH}_{2} \mathrm{Cl}_{2}$ thoroughly to afford the pure product.

(-)-(2S)-I-(N-diphenyIphosphinyl)aziridin-2-yI(diphenyI)methanol IIIa:

white solid m.p. $>240^{\circ} \mathrm{C},[]_{\mathrm{D}}{ }^{20}-26.0$ (c 0.27 , DMF), ${ }^{31} \mathrm{P}$ NMR $32.72 \mathrm{ppm},{ }^{1} \mathrm{H}$ NMR $2.40 \sim 2.80(\mathrm{~m}, 2 \mathrm{H}$, $\left.\mathrm{CH}_{2}\right), 3.10$ (bs, $\left.1 \mathrm{H}, \mathrm{OH}\right), 3.85 \sim 3.90(\mathrm{~m}, 1 \mathrm{H}, \mathrm{CH}), 6.95 \sim 7.95(\mathrm{~m}, 20 \mathrm{H}, \mathrm{Ph}-\mathrm{H})$. Anal. Calcd for $\mathrm{C}_{27} \mathrm{H}_{22} \mathrm{NO}_{2} \mathrm{P}$ : C, 76.22; H, 5.69; N, 3.29. Found: C, 76.10; H, 5.50; N, 3.35.

(-)-(2S)-I -(N-diphenylphosphinyl)aziridin-2-yI|di(p-methyl)-phenyl|methanol IIIb:

white solid m.p. $>240^{\circ} \mathrm{C},[]_{\mathrm{D}}^{20}+4.0\left(\mathrm{c} 3.10, \mathrm{CHCl}_{3}\right)$, ${ }^{2} \mathrm{P}$ NMR $26.98 \mathrm{ppm},{ }^{1} \mathrm{H}$ NMR $2.25 \sim 2.31(\mathrm{~d}, 6 \mathrm{H}$, $\left.2 \mathrm{CH}_{3}\right), 3.10 \sim 3.32\left(\mathrm{~m}, 3 \mathrm{H}, \mathrm{CH}_{2}, \mathrm{OH}\right), 5.05 \sim 5.10(\mathrm{t}, \mathrm{HH}, \mathrm{CH}), 7.06 \sim 7.85(\mathrm{~m}, 18 \mathrm{H}, \mathrm{Ph}-\mathrm{H})$. Anal. Calcd for $\mathrm{C}_{29} \mathrm{H}_{28} \mathrm{NO}_{2} \mathrm{P}: \mathrm{C}, 76.80 ; \mathrm{H}, 6.22 ; \mathrm{N}, 3.09$. Found: $\mathrm{C}, 76.62 ; \mathrm{H}, 6.16 ; \mathrm{N}, 3.21$.

(-)-(2S)-I-(N-diphenylphosphinyl)aziridin-2-yI[di(p-fluoro)-phenyl|methanol IIIc:

white solid m.p. $>240^{\circ} \mathrm{C}$, [] ${ }_{\mathrm{D}}^{20}-17.8\left(\mathrm{c} 0.90, \mathrm{CHCl}_{3}\right),{ }^{31} \mathrm{P}$ NMR $32.85 \mathrm{ppm},{ }^{\mathrm{H}} \mathrm{H}$ NMR $2.40 \sim 2.60(\mathrm{~m}$, $1 \mathrm{H}, \mathrm{H}), 3.50 \sim 3.80(\mathrm{~m}, 2 \mathrm{H}, \mathrm{H}, \mathrm{OH}), 6.57 \sim 6.60(\mathrm{t}, 1 \mathrm{H}, \mathrm{CH}), 6.93 \sim 7.48(\mathrm{~m}, 18 \mathrm{H}, \mathrm{Ph}-\mathrm{H})$. Anal. Calcd for $\mathrm{C}_{27} \mathrm{H}_{22} \mathrm{~F}_{2} \mathrm{NO}_{2} \mathrm{P}: \mathrm{C}, 70.28 ; \mathrm{H}, 4.81 ; \mathrm{N}, 3.04$. Found: $\mathrm{C}, 69.98 ; \mathrm{H}, 4.76 ; \mathrm{N}, 3.15$.

Typical procedure for the asymmetric reduction of prochiral ketones catalyzed by I III:

To a solution of $2 \mathrm{ml}$ toluene and $10 \mathrm{~mol} \%$ I III, was added $1.2 \mathrm{eq} \mathrm{BH}_{3} \mathrm{Me}_{2} \mathrm{~S}$ under nitrogen atmosphere. The mixture was stirred for $20 \mathrm{~min}$, then a solution of $1 \mathrm{mmol}$ prochiral ketone in $4 \mathrm{ml}$ dry toluene was added in 30 mins. The reaction was complete (monitored by TLC) after the addition. $10 \mathrm{ml}$ saturated $\mathrm{NH}_{4} \mathrm{Cl}$ solution was added and the resulting mixture was stirred for another 10 mins. The organic phase was separated and the water layer was extrated with $210 \mathrm{ml}$ EtOAc. The combined organic layer was washed with saturated $\mathrm{NaCl}$ solution and dried over anhydrous $\mathrm{Na}_{2} \mathrm{SO}_{4}$.

\section{Scheme 4}
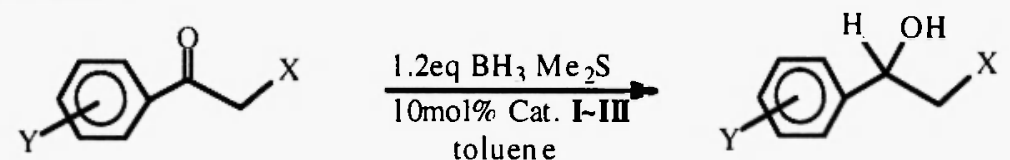

Table I Asymmetric borane reduction of prochiral ketones catalyzed by I III

\begin{tabular}{|c|c|c|c|c|c|c|c|}
\hline Cat. & $\mathrm{X}$ & $\mathrm{Y}$ & $\mathrm{Temp} . /{ }^{\circ} \mathrm{C}$ & $\mathrm{Yield} / \% \%^{\mathrm{a}}$ & {$\left[\mathrm{D}^{25}{ }^{\mathrm{c}}\right.$} & $\mathrm{e} . \mathrm{e} / \% \%^{\mathrm{d}}\left(\mathrm{ee} / \%{ }^{\mathrm{e}}\right)$ & Config. \\
\hline I & $\mathrm{Cl}$ & $\mathrm{H}$ & $-20 \sim \mathrm{rt}$ & 88.7 & -30.0 & $63.8(62.4)$ & $\mathrm{S}$ \\
\hline I & $\mathrm{Cl}$ & $\mathrm{H}$ & $30 \sim 40$ & 95.2 & -26.0 & $55.5(54.2)$ & $\mathrm{S}$ \\
\hline I & $\mathrm{Cl}$ & $\mathrm{H}$ & $60 \sim 70$ & 93.5 & -24.3 & $51.7(49.8)$ & $\mathrm{S}$ \\
\hline I & $\mathrm{Cl}$ & $\mathrm{H}$ & Reflux & 92.7 & - & 11.1 & $\mathrm{~S}$ \\
\hline I & $\mathrm{Br}$ & $\mathrm{H}$ & $30 \sim 40$ & 94.3 & -28.3 & 50.7 & $\mathrm{~S}$ \\
\hline I & $\mathrm{H}$ & $\mathrm{H}$ & $-20 \sim \mathrm{rt}$ & 91.7 & -21.5 & 45.8 & $\mathrm{~S}$ \\
\hline II & $\mathrm{Cl}$ & $\mathrm{H}$ & $30 \sim 40$ & 96.8 & +20.0 & 46.5 & $\mathrm{R}$ \\
\hline II & $\mathrm{H}$ & $\mathrm{H}$ & $30 \sim 40$ & 83.0 & +15.0 & 33.3 & $\mathrm{R}$ \\
\hline II & $\mathrm{H}$ & $\mathrm{o}-\mathrm{Cl}$ & $30 \sim 40$ & 88.7 & +10.1 & 15.4 & $\mathrm{R}$ \\
\hline II & $\mathrm{H}$ & $\mathrm{p}-\mathrm{Me}$ & $30 \sim 40$ & 93.5 & +29.0 & 50.3 & $\mathrm{R}$ \\
\hline II & $\mathrm{H}$ & $\mathrm{p}-\mathrm{MeO}$ & $30 \sim 40$ & 91.7 & +17.5 & 54.2 & $\mathrm{R}$ \\
\hline IIIa & $\mathrm{Cl}$ & $\mathrm{H}$ & Reflux & 92.7 & +23.9 & 54.5 & $\mathrm{R}$ \\
\hline IIIa & $\mathrm{H}$ & $\mathrm{p}-\mathrm{MeO}$ & Reflux & 92.0 & +14.5 & 44.9 & $\mathrm{R}$ \\
\hline IIIa & $\mathrm{H}$ & $\mathrm{p}-\mathrm{Me}$ & Reflux & 92.0 & +5.0 & 8.7 & $\mathrm{R}$ \\
\hline IIIa & $\mathrm{H}$ & $\mathrm{o}-\mathrm{Cl}$ & Reflux & 90.0 & 0 & 0 & - \\
\hline IIIb & $\mathrm{Cl}$ & $\mathrm{H}$ & Reflux & 92.5 & +4.3 & 10.0 & $\mathrm{R}$ \\
\hline IIIb & $\mathrm{H}$ & $\mathrm{p}-\mathrm{MeO}$ & Reflux & 95.8 & +17.0 & 52.6 & $\mathrm{R}$ \\
\hline IIIb & $\mathrm{H}$ & $\mathrm{p}-\mathrm{Me}$ & Reflux & 84.0 & +6.6 & 11.4 & $\mathrm{R}$ \\
\hline IIIb & $\mathrm{H}$ & $\mathrm{o}-\mathrm{Cl}$ & Reflux & 92.7 & +4.3 & 6.6 & $\mathrm{R}$ \\
\hline IIIc & $\mathrm{Cl}$ & $\mathrm{H}$ & Reflux & 88.3 & +7.0 & 16.0 & $\mathrm{R}$ \\
\hline IIIc & $\mathrm{H}$ & $\mathrm{p}-\mathrm{MeO}$ & Reflux & 96.7 & +2.0 & 6.0 & $\mathrm{R}$ \\
\hline
\end{tabular}

a. isolated yield; $b$. $c=1.0$; c.determined by specific rotation, for $(\mathrm{S})-1$-phenylethanol $\int_{D^{20}}{ }^{2}=-45.5$ (c 2.0 , $\mathrm{MeOH})^{[\mathrm{sd}]}$, for (R)-2-Chloro-1-phenylethanol []$_{\mathrm{D}}^{20}=+47$ (c 1.84 , cyclohexane $)^{i \mathrm{iv}}$, for $(\mathrm{R})-1-(2-$ Chlorophenyl)ethanol []$_{\mathrm{D}}^{20}=+65.7\left(\mathrm{c} 0.625, \mathrm{CHCl}_{3}\right)^{(11]}$, for (R)-1-(2-Bromophenyl)ethanol $\left[\mathrm{D}^{20}=+58.8(\mathrm{c}\right.$ $\left.0.57, \mathrm{CH}_{2} \mathrm{Cl}_{2}\right)^{[12]}$, for $(\mathrm{R})-1-\left(4-\right.$ Methoxyphenyl)ethanol []$_{\mathrm{D}}{ }^{20}=+32.3\left(\mathrm{c} 2.0, \mathrm{CHCl}_{3}\right)^{[1.1]}$, for $\mathrm{I}-(4-$ Methylphenyl)ethanol []$_{D}=+57.5\left(\mathrm{c} 0.36, \mathrm{CHCl}_{3}\right)^{[14]}$; d. determined Chiral HPLC analysis. 
After removal of the solvent the crude product was purified by column chromatography on silica gel. The catalyst could be recycled and reused. The enantioselective excess of the product could be determined by the comparasion the specific rotation with literature or by chiral HPLC analysis.

\section{RESULTS AND DISCUSSION}

The prepared chiral phosphinamides I III were used as catalysts in the borane reduction of prochiral ketones. (Scheme 4). The results are summarized in Table 1.

As shown in Table 1, in all cases, the chiral secondary alcohols were obtained with excellent chemical yields and moderate enantiomeric excesses. The reaction temperature had a marked influence on the enantioselectivity in the reduction of 2-chloro-1-phenylethanone catalyzed by I. In general, lower temperature was beneficial for the improvement of the enantioselectivity. For example, carring out the reaction at $-20^{\circ} \mathrm{C}$ provided the optical product with $63.8 \%$ ee value which was much higher than the results obtained at $30 \sim 40^{\circ} \mathrm{C}\left(55.5 \%\right.$ ee) and $60 \sim 70^{\circ} \mathrm{C}(51.7 \%$ ee), respectively. The ee value dramatically decreased to $11.1 \%$ at reflux temperature. To the other two substrates, 2-bromo-1-phenylethanone and acetophenone, only moderate ee were also obtained at definite condition. For catalyst II, the nature of the substrates was found to be an essential factor to the asymmetric induction. Compared with acetophenone ( $33.3 \%$ ee), much higher catalytic activities ( $50.3 \%$ ee and $54.2 \%$ ee respectively) were observed when there was electrondonor group (Me-, MeO-) substituted at the para-positon of the benzene ring in acetophenone. On the contrary, the enantiomeric excess decreased rapidly $(15.4 \% \mathrm{ee})$ when electron-withdrawing group, such as $\mathrm{Cl}$, was present instead. Our further study demonstrated that only low enantiomeric excesses ( $0 \sim 54.5 \%$ ee) were obtained when 11Ia c were employed in the reduction of a series of prochiral ketones. A possible interpret is that the large strain of the three-membered ring in catalysts IIIa c led to the low catalytic activities but it still needs to be proved with more experimental data.

In conclusion, a new class of chiral phosphinamide catalysts was synthesized and their application as catalyst in the reduction of prochiral ketone were investigated. The corresponding alcohols were obtained in excellent chemical yields with moderate ee-values. The impact factors on the selectivities were also studied. These findings will give us very useful information for the design and synthesis of the new types of chiral catalysts in our ongoing research program.

\section{ACKNOWLEDGEMENT}

We are very grateful for the financial support of the National Natural Science Foundation of China (No. 20002002 and No.20272025)

\section{REFERENCES}

1. a. Noyori, R., Asymmetric Catalysis in Organic Synthesis, Wiley, New York, 1994.

b. Ojima, l., ed, Catalytic Asymmetric Synthesis, VCH Press, Berlin, 1993.

c. Singh, V. K. Synthesis, 1992, 605.

d. Brown, H. C., Ramachandran, P. V. Acc. Chem. Res, 1992, 25(1), 16.

e. Noyori, R. Chem. Soc. Rev., 1989, 18, 187.

1. a. Togni, A.; Venanzi, L. M. Angew. Chem., Int. Ed. 1994, 33, 497.

b. Deloux, L.; Srebnik, M. Chem. Rev. 1993, 93, 763.

2. a. Corey, E. J., Helal, C. J. Angew Chem., Int. Ed. Engl. 1998, 37, 1987.

b. Schunicht, C., Biffis, A., Wulff, G. Tetrahedron 2000, 56, 6041.

3. Bolm, C., Felder, M. Tetrahedron Lett. 1993, 34(38), 6041.

4. a. Wills, M., Gamble, M., Palmer, M., Smith, A., Studley, J. R., Kenny, J. J. Molec. Cat, A: Chem. 1999, $146,139$.

b. Buono, G., Chiodi, O., Wills, M. Synlett 1999, (4), 377.

c. Burns, B.; Studley, J. R.; Wills, M. Tetrahedron Lett. 1993, 34(44), 7105.

d. Burns, B.; King, N. P.; Tye, H.; Wills, M. Tetrahedron: Asymmetry 1994, 5(5), 801.

e. Chiodi, O.; Fotiadu, F.; Sylvestre, M.; Buono, G. Tetrahedron Lett. 1996, 37(1), 39.

f. Gamble, M. P.; Studley, J. R.; Wills, M. Tetrahedron: Asymmetry 1996, 7(11), 3071.

g. Gamble, M. P.; Studley, J. R.; Wills, M. Tetrahedron Lett. 1996, 37(16), 2853.

h. Peper, V.; Martens, J. Tetrahedron Lett. 1996, 37(46), 8351.

i. Hulst, R.; Heres, H.; Peper, N. C. M. W.; Kellogg, R. M. Tetrahedron: Asymmetry 1996, 7(5), 1373.

j. Brunel, J-M.; Chiodi, O.; Faure, B.; Fotiadu, F.; Buono, G. J. Organometal. Chem. 1997, 529, 285.

k. Gamble, M. P.; Smith, A. R. C.; Wills, M. J. Org. Chem. 1998, 63(17), 6068.

I. Burns, B.; King, N. P.; Tye, H.; Studley, J. R.; Gamble, M.; wills, M. J. Chem. Soc., Perkin Trans. I, $1998,1027$.

m. Chen, G-H; Hsu, J.-L.; Yan, W.-B.; Fang, J.-M.; Lee, G.-H.; Liu, Y.-H.; Wang, Y.; J. Chinese Chem. Soc. $1999,46,797$.

n. Brunnel, J.M.; Legrand, O.; Buono, G. Eur. J. Org. Chem. 2000, 19, 3313. 
5. Betti, M. Organic Syntheses Coll. 1941, 1, 381.

6. Fischer, F.; Tiedt, H-J.; Wolf, K.; Platz, K-H. J. Prakt. Chem. 1965, 4(28), 157.

7. Baldwin, J. E.; Spivey, A. C.; Schofield, C. J.; Sweeney, J. B. Tetrahedron, 1993, 49(28), 6309.

8. Willems, J. G. H.; Hersmis, M. C.; Gelder, R. de.; Smits, J. M. M.; Hammink, J. B.; Dommerholt, F. J.; Thijs, L.; Zwanenburg, B. J. Chem. Soc., Perkin Trans. I, 1997, 963.

9. Wilken, J.; Martens, J. Synth.Commun. 1996, 26, 4477.

10. Nakamura, K.; Kawasaki, M.; Ohno, A. Bull. Chem. Soc. Jpn. 1996, 69. 1079.

11. Resnick, S. M.; Torok, D. S., Gibson, D. T. J. Org. Chem. 1995, 60, 3546.

12. Brown, S. M.; Davis, S. G.: de Sousa, J. A. A. Tetrahedron Asymmetry 1993, 4, 813.

13. Nakamura, K.; Kawasaki, M.; Ohno, A. Bull. Chem. Soc. Jpn. 1996, 69, 1079.

Received: September 30, 2002 - Accepted: October 4, 2002 Accepted in publishable format: October 7, 2002 
